\title{
Phase I clinical study of anti-apoptosis protein, survivin-derived peptide vaccine therapy for patients with advanced or recurrent colorectal cancer
}

\author{
Tetsuhiro Tsuruma*1, Fumitake Hata1, Toshihiko Torigoe ${ }^{2}$, \\ Tomohisa Furuhata1, Satomi Idenoue ${ }^{1}$, Takehiro Kurotaki1,2, \\ Masaaki Yamamoto ${ }^{1,2}$, Atsuhito Yagihashi ${ }^{3}$, Tosei Ohmura ${ }^{1}$, \\ Koji Yamaguchi ${ }^{1}$, Tadashi Katsuramaki ${ }^{1}$, Takahiro Yasoshima ${ }^{4}$, \\ Kazuaki Sasaki ${ }^{5}$, Yasuhiro Mizushima ${ }^{6}$, Hidetoshi Minamida7, \\ Hiromichi Kimura8, Morifumi Akiyama9, Yoshihiko Hirohashi², \\ Hiroko Asanuma ${ }^{2}$, Yasuaki Tamura ${ }^{2}$, Kumiko Shimozawa ${ }^{10}$, Noriyuki Sato ${ }^{2}$ \\ and Koichi Hirata ${ }^{1}$
}

\begin{abstract}
Address: ${ }^{1}$ Department of Surgery, Sapporo Medical University School of Medicine, Sapporo, Japan, ${ }^{2}$ Department of Pathology, Sapporo Medical University School of Medicine, Sapporo, Japan, ${ }^{3}$ Department of Laboratory Diagnosis, Sapporo Medical University School of Medicine, Sapporo, Japan, ${ }^{4}$ Department of Surgery, Shinsapporo Keiaikai Hospital, Sapporo, Japan, ${ }^{5}$ Department of Surgery, Doto Hospital, Sapporo, Japan, ${ }^{6}$ Department of Surgery, Ashibetsu Municipal Hospital, Ashibetsu, Japan, ${ }^{7}$ Department of Surgery, Chitose Daiichi Hospital, Chitose, Japan, ${ }^{8}$ Department of Surgery, Sapporo Tsukisamu Hospital, Sapporo, Japan, ${ }^{9}$ Department of Surgery, Higashi Sapporo Hospital, Sapporo, Japan and ${ }^{10}$ Japan Science and Technology Corporation Innovation Plaza Hokkaido, Sapporo, Japan

Email: Tetsuhiro Tsuruma* - tsuruma@sapmed.ac.jp; Fumitake Hata - fhata@sapmed.ac.jp; Toshihiko Torigoe - torigoe@sapmed.ac.jp; Tomohisa Furuhata - furuhata@sapmed.ac.jp; Satomi Idenoue - idenoue@sapmed.ac.jp; Takehiro Kurotaki - kurokuro@sea.plala.or.jp; Masaaki Yamamoto - masa@sapmed.ac.jp; Atsuhito Yagihashi - yagihasi@sapmed.ac.jp; Tosei Ohmura - ohmura@sapmed.ac.jp; Koji Yamaguchi - yamakoji@sapmed.ac.jp; Tadashi Katsuramaki - katsuramaki@sapmed.ac.jp; Takahiro Yasoshima - yasosima@sapmed.ac.jp; Kazuaki Sasaki - kazuaki@doto.org; Yasuhiro Mizushima - nomura@sapmed.ac.jp; Hidetoshi Minamida - ogami-m@xpost.plala.or.jp; Hiromichi Kimura - hkimura@sapmed.ac.jp; Morifumi Akiyama - yajima@sapmed.ac.jp; Yoshihiko Hirohashi - yhirohashi@yahoo.co.jp; Hiroko Asanuma - asanuma@sapmed.ac.jp; Yasuaki Tamura - ytamura@sapmed.ac.jp; Kumiko Shimozawa - simozawa@sapmed.ac.jp; Noriyuki Sato - nsatou@sapmed.ac.jp; Koichi Hirata - jtakagi@sapmed.ac.jp

* Corresponding author
\end{abstract}

Published: 13 June 2004

Journal of Translational Medicine 2004, 2:19 doi:10.1 186/1479-5876-2-19
Received: 02 April 2004

Accepted: 13 June 2004

This article is available from: http://www.translational-medicine.com/content/2/1/19

(C) 2004 Tsuruma et al; licensee BioMed Central Ltd. This is an Open Access article: verbatim copying and redistribution of this article are permitted in all media for any purpose, provided this notice is preserved along with the article's original URL.

\begin{abstract}
Survivin is a member of the inhibitor of apoptosis protein (IAP) family containing a single baculovirus IAP repeat domain. It is expressed during fetal development but becomes undetectable in terminally differentiated normal adult tissues. We previously reported that survivin and its splicing variant survivin-2B was expressed abundantly in various types of tumor tissues as well as tumor cell lines and was suitable as a target antigen for active-specific anti-cancer immunization. Subsequently, we identified an HLA-A24-restricted antigenic peptide, survivin-2B80-88 (AYACNTSTL) recognized by CD8+ cytotoxic T lymphocytes (CTLs). We, therefore, started a phase I clinical study assessing the efficacy of survivin-2B peptide vaccination in patients with advanced or recurrent colorectal cancer expressing survivin. Vaccinations with survivin-2B peptide were given subcutaneously six times at 14-day intervals. Of 15 patients who finished receiving the
\end{abstract}


vaccination schedule, three suffered slight toxicities, including anemia (grade 2), general malaise (grade I), and fever (grade I). No severe adverse events were observed in any patient. In 6 patients, tumor marker levels (CEA and CA19-9) decreased transiently during the period of vaccination. Slight reduction of the tumor volume was observed in one patient, which was considered a minor responder. No changes were noted in three patients while the remaining eleven patients experienced tumor progression. Analysis of peripheral blood lymphocytes of one patient using HLA-A24/peptide tetramers revealed an increase in peptide-specific CTL frequency from $0.09 \%$ to $0.35 \%$ of CD8+ $\mathrm{T}$ cells after 4 vaccinations. This phase I clinical study indicates that survivin-2B peptide-based vaccination is safe and should be further considered for potential immune and clinical efficacy in HLA-A24-expression patients with colorectal cancer.

\section{Background}

Most patients who undergo operations for advanced colorectal cancer remain at high risk for local or systemic recurrence, and current chemotherapy or radiotherapy regimens have limited efficacy in preventing recurrence. Hence, advances in new therapeutic modalities for patients with advanced or recurrent colorectal cancer are urgently needed, one of which is tumor-specific immunotherapy.

Tumor cells express antigens that can be recognized by the host's immune system. T cells recognize antigens in the form of short peptides binding to major histocompatibility complex (MHC) molecules [1]. Following the first report of the cloning of a human tumor antigen gene, termed melanoma antigen-1 (MAGE-1) in 1991 [2], a nonapeptide encoded by this gene was identified that could be recognized by HLA-A1-restricted CTLs [3]. To date, a large number of antigenic peptides have been identified from melanoma and other cancers, and clinical trials of peptide-based immunotherapy for cancer have taken place. Melanoma antigen peptides were the first to be tested in phase I and phase II studies for active immunization of metastatic melanoma patients $[2,4]$. In some studies, clinical responses have been observed in 10-30\% of the patients treated [5]. Rosenberg et al. reported that clinical responses were observed in $42 \%$ of patients with metastatic melanoma receiving a modified gp 100 peptide (gp100-209.2M) and systemic high dose of IL-2 [6]. Since then, various cancer vaccine trials are ongoing in the United States and Europe [5].

Survivin was identified as a member of the inhibitor of apoptosis protein (IAP) family with a single baculovirus IAP repeat domain [7]. Survivin is present during fetal development but undetectable in terminally differentiated normal adult tissues except for certain cells such as proliferating endothelial cells and activated lymphocytes. In contrast to normal tissues, survivin is abundantly expressed in transformed cell lines and in most common cancers, including colorectal cancer [8]. We previously reported that survivin-2B, a splicing variant of survivin was also expressed in various types of tumor cell lines ${ }^{9}$, and the survivin-2B80-88 (AYACNTSTL) peptide derived from exon 2B-encoded region was recognized by CD8+ CTLs in the context of HLA-A24 molecules [9]. CTLs specific for this peptide were successfully induced from peripheral blood mononuclear cells (PBMCs) in 6 out of 7 HLA-A24+ patients (83\%) with colorectal cancers and exerted cytotoxicity against HLA-A24+ / survivin+ adenocarcinoma cells [10]. On the basis of these observations, we have started a phase I clinical study of survivin-2B peptide vaccination for patients with advanced or recurrent colorectal cancer. Our present study demonstrated safety and suggested marginal clinical effectiveness of the survivin-2B peptide vaccination in colorectal cancer patients.

\section{Methods \\ Patient selection}

The study protocol was approved by the Clinic Institutional Ethical Review Board of the Medical Institute of Bioregulation, Sapporo Medical University, Japan. All patients gave informed consent before being enrolled. Patients enrolled in this study were required to conform to the following criteria: (1) to have histologically confirmed colorectal cancer, (2) to be HLA-A*2402 positive, (3) to be survivin-positive in the carcinomatous lesions by immunohistochemistry, (4) to be between 20 and 85 years old, (5) to have received surgical excision of the primary with curative intent and (6) to have ECOG performance status between 0 and 3. Exclusion criteria included (1) prior cancer therapy, such as chemotherapy, radiation therapy, steroid therapy, or other immunotherapy within the past 4 weeks, (2) presence of other cancers that might influence the prognosis, (3) immunodeficiency or a history of splenectomy, (4) severe cardiac insufficiency, acute infection, or hematopoietic failure (5) ongoing breastfeeding, (6) unsuitability for the trial based on clinical judgment. This study was carried out at the Department of Surgery, Sapporo Medical University Primary Hospital from March 2003 through December 2003. 


\section{Peptides preparation}

The peptide, survivin-2B80-88 with the sequence AYACNTSTL, was prepared under good manufacturing practice conditions by Multiple Peptide Systems (San Diego, CA). The identity of the peptide was confirmed by mass spectral analysis, and the purity was shown to be more than $98 \%$ as assessed by high pressure liquid chromatography analysis.

The peptide was supplied as a freeze-dried, sterile white powder. It was dissolved in $1.0 \mathrm{ml}$ of physiological saline (Otsuka Pharmaceutical Co., Ltd., Tokyo, Japan) and stored at $-80^{\circ} \mathrm{C}$ until just before use.

\section{Patient treatment}

The vaccination schedules were as follows. Vaccinations with survivin-2B peptide were given subcutaneously (s.c.) six times at 14-day intervals. In order to set up a dose-escalation trial, the patients were separated into the following three groups: group 1 patients received $0.1 \mathrm{mg}$, group 2 received $1.0 \mathrm{mg}$ and group 3 received $10 \mathrm{mg}$. Each group included five patients. Escalation to the next dose was allowed if side effects did not exceed grade 3 .

\section{Delayed-type hypersensitivity (DTH) skin test}

Delayed-type hypersensitivity (DTH) skin test was performed at each vaccination. The peptide $(10 \mu \mathrm{g})$ solution in physiological saline $(0.1 \mathrm{ml})$ or physiological saline alone $(0.1 \mathrm{ml})$ were separately injected intradermally (i.d.) into the forearm opposite to the vaccination site. A positive reaction was defined as a more than $4 \mathrm{~mm}$ diameter area of erythema and induration $48 \mathrm{hr}$ after the injection.

\section{Toxicity evaluation}

Patients were examined closely for signs of toxicity during and after vaccination. Adverse events were recorded using the National Cancer Institute Common Toxicity Criteria (NCI-CTC).

\section{Clinical response evaluation}

Physical examinations and hematological examinations were monitored before and after each vaccination. Tumor markers (CEA and CA19-9) were examined monthly. Tumor size was evaluated by computed tomography (CT) scans or MRI before treatment, after three vaccinations and at the end of the study period. A complete response (CR) was defined as complete disappearance of all measurable and evaluable disease. A partial response (PR) was defined as $\mathrm{a}>=50 \%$ decrease from the baseline in size of all measurable lesions (sum of products of maximal perpendicular diameters) lasting at least 4 weeks. Progressive disease (PD) was defined as an increase in the sum of the bi-dimensional measurements of all known disease sites by at least $25 \%$ or the appearance of new lesions. No change (NC) was defined as the absence of matched criteria for $C R, P R$, or $P D$. In addition, one case that was defined as NC but reached a nearly PR, was recorded as minor response $(\mathrm{MR})$. Therefore, a MR was defined as > $50 \%$ decrease from the baseline in all measurable lesions (sum of products of maximal perpendicular diameters) lasting less than 4 weeks or as a $25 \%$ to $50 \%$ decrease from the baseline in all measurable lesions (sum of products of maximal perpendicular diameters) lasting at least 4 weeks. Patients who received less than six vaccinations were excluded from all evaluations in this study.

\section{Tetramer staining}

HLA-A24/peptide tetramers were constructed according to the procedure described by Altman et al. [11]. Briefly, recombinant HLA-A24 heavy chain [12] and human $\beta$-2microgloblin were refolded with survivin-2B80-88 peptides as described before [13]. The resulting HLA-A24peptide monomer was biotinylated by incubation with BirA enzyme (Avidity, Denver, CO) for $17 \mathrm{hr}$ at room temperature and purified using fast protein liquid chromatography. Tetrameric HLA-peptide complex was produced by incubating streptavidin-PE (Vector Laboratories, Burlingame, CA) with the biotinylated monomer at a 1:4 molar ratio. For flow cytometric analysis, PBMCs were isolated from blood samples by Ficoll-Conray density gradient centrifugation, and stained with PE-labeled tetramer at $37^{\circ} \mathrm{C}$ for $20 \mathrm{~min}$, followed by staining with FITC-conjugated anti-CD8 mAb (Beckton Dickinson Biosciences) at $4^{\circ} \mathrm{C}$ for $30 \mathrm{~min}$. Cells were washed twice with PBS before fixation in $1 \%$ formaldehyde. Flow cytometric analysis was performed using FACSCalibur and Cell Quest software (BD Biosciences). The frequency of CTL precursors was calculated as the number of tetramer-positive cells over the number of CD8-positive cells.

\section{ELISPOT assay}

ELISPOT plates were coated sterilely overnight with IFN- $\gamma$ capture antibody (Beckton Dickinson Biosciences) at $4^{\circ} \mathrm{C}$. The plates were washed once and blocked with AIM$\mathrm{V}$ medium containing 10\% human serum for $2 \mathrm{hr}$ at room temperature. CD8-positive T cells separated from patients' PBMCs $\left(5 \times 10^{3}\right.$ cells/well $)$ were then added to each well along with HLA-A24-transfected CIR cells (CIR-A24) $(5 \times$ $10^{4}$ cells/well), which had been pre-incubated with or without survivin-2B80-88 peptides $(10 \mu \mathrm{g} / \mathrm{ml})$. After incubation in a $5 \% \mathrm{CO}_{2}$ humidified chamber at $37^{\circ} \mathrm{C}$ for 24 hours, the wells were washed vigorously five times with PBS and incubated with biotinylated anti-human IFN- $\gamma$ antibody and horseradish peroxidase-conjugated avidin. Spots were visualized and analyzed by KS ELISPOT (Carl Zeiss, Germany). 
Table I: Profiles of patients enrolled in this study and clinical responses to the vaccination

\begin{tabular}{|c|c|c|c|c|c|c|c|}
\hline patient no. & age & sex & $\begin{array}{c}\text { dose of } \\
\text { peptide }(\mathrm{mg})\end{array}$ & $\begin{array}{l}\text { adverse } \\
\text { events }\end{array}$ & $\begin{array}{l}\text { tumor } \\
\text { marker }\end{array}$ & $\begin{array}{l}\text { evaluation of } \\
\text { CT image }\end{array}$ & DTH skin test \\
\hline 1 & 63 & $M$ & 0.1 & anemia & decreased & $M R$ & -- \\
\hline 2 & 53 & $M$ & 0.1 & -- & not decreased & PD & -- \\
\hline 3 & 42 & $M$ & 0.1 & -- & decreased & $\mathrm{NC}$ & -- \\
\hline 4 & 54 & $\mathrm{~F}$ & 0.1 & -- & & & \\
\hline 5 & 65 & $M$ & 0.1 & -- & decreased & PD & -- \\
\hline 6 & 64 & $\mathrm{~F}$ & 0.1 & general malaise & decreased & PD & + \\
\hline 7 & 48 & $\mathrm{~F}$ & 1.0 & -- & decreased & PD & -- \\
\hline 8 & 62 & $\mathrm{~F}$ & 1.0 & -- & not decreased & PD & -- \\
\hline 9 & 64 & $M$ & 1.0 & -- & decreased & PD & -- \\
\hline 10 & 52 & $M$ & 1.0 & -- & not decreased & PD & + \\
\hline 11 & 58 & $M$ & 1.0 & -- & not decreased & NC & + \\
\hline 12 & 58 & $\mathrm{~F}$ & 10.0 & fever & not decreased & PD & -- \\
\hline 13 & 62 & $\mathrm{~F}$ & 10.0 & -- & not decreased & PD & + \\
\hline 14 & 72 & $M$ & 10.0 & -- & not decreased & PD & + \\
\hline 15 & 71 & $M$ & 10.0 & -- & & & \\
\hline 16 & 67 & $M$ & 10.0 & -- & not decreased & PD & -- \\
\hline 17 & 59 & $\mathrm{~F}$ & 10.0 & -- & not decreased & NC & + \\
\hline
\end{tabular}

a) MR: minor response, NC: no change, PD: progressive disease

\section{Results \\ Patient profiles}

Seventeen patients were initially enrolled in the study (Table 1), but two (case 4 and 15) discontinued halfway through the protocol. One patient (case 4) had lung and brain metastases from rectal cancer and was removed from the study after 2 vaccinations since he required steroid therapy to suppress cerebral edema resulting from brain metastasis. Another patient (case 15) had lung metastases, liver metastases and carcinomatosis from colon cancer. He was removed from the study after 4 vaccinations because of exacerbated dyspnea resulting from advanced pulmonary metastases. Neither of the treatment interruptions were due to the adverse effects of the vaccination. Fifteen patients received the complete regimen including six vaccinations and were evaluated. They included 9 men and 6 women, whose average age was 59 years (range, 42-72). In for 4 cases colon cancer was the primary diagnosis and the remaining 11 cases resulted from rectal cancer.

\section{Safety}

Peptide vaccination was well tolerated in all 15 patients. Three patients (case 1, 6, and 12) had slight toxicity-associated symptoms such as anemia (grade 2), general malaise (grade 1) and fever (grade 1). Case 1 developed anemia after the second vaccination. Case 6 and case 12 experienced general malaise and fever after each vaccination followed by spontaneous recovery within a few days. No serious adverse events were observed during or after vaccination.

\section{Clinical response}

Table 1 summarizes the clinical outcomes for the 15 patients. In 6 patients (case 1 , case 3 , case 5 , case 6 , case 7 , case 9) tumor marker levels (CEA and CA19-9) transiently decreased or at least did not increase during vaccination (Table 2). A minor response was observed in one patient (case 1) who also demonstrated a decrease in CEA level. Three patients (case 3, 11, 17) were considered NC and the remaining 11 PD.

It was noted retrospectively that all six patients in whom a decrease in tumor marker level was observed had received some sort of adjuvant chemotherapy after resection of the primary tumor without additional chemotherapy after recurrence (Fig. 1). The details are as follows: case 1 has received a daily dose of $600 \mathrm{mg}$ tegafur/uracil (UFT) for eight months as oral adjuvant chemotherapy. He stopped receiving the drug at his own request. Case 3 received tegafur/uracil (UFT) at a daily dose of $600 \mathrm{mg}$ for two years as oral adjuvant chemotherapy. He discontinued the medicine due to severe nausea. Case 5 received 5 FU at a daily dose of $200 \mathrm{mg}$ for three years as oral adjuvant chemotherapy. Case 6 received 2 courses of CPT-11 ( $40 \mathrm{mg} /$ day on the day 1, 8 and 15) every 4 weeks as adjuvant chemotherapy. Subsequently, the patient discontinued the treatment because of an increase in tumor markers and a recurrence in the pleural cavity. Case 7 received 2 courses of leucovorin $(750 \mathrm{mg} /$ day on the day $1,8$ and 15$)$ and 5-FU (250 mg/day on the day 1, 8 and $15)$ every 4 weeks as adjuvant chemotherapy. The patient discontinued the treatment due to toxic effects that 
Table 2: CEA levels of 6 patients who showed temporary decrease during the vaccination

\begin{tabular}{|c|c|c|c|c|}
\hline Patient & Baseline level (weeks) $* 1 * 2$ & Lowest level (weeks) $* 2$ & Percent reduction $* 3$ & $\begin{array}{l}\text { Duration of the } \\
\text { decrease in CEA }\end{array}$ \\
\hline Case I & 15.9 ng/ml (0 week) & 6.5 ng/ml (7 weeks) & $59.1 \%$ & 12 weeks \\
\hline Case 3 & $34.0 \mathrm{ng} / \mathrm{ml}$ (5 weeks) & $30.4 \mathrm{ng} / \mathrm{ml}$ (9 weeks) & $10.6 \%$ & 6.5 weeks \\
\hline Case 5 & $184.0 \mathrm{ng} / \mathrm{ml}(0$ week) & $159.2 \mathrm{ng} / \mathrm{ml}$ (2 weeks) & $13.5 \%$ & 8 weeks \\
\hline Case 6 & $19.5 \mathrm{ng} / \mathrm{ml}$ (8 weeks) & $19.4 \mathrm{ng} / \mathrm{ml}$ (10 weeks) & $0.5 \%$ & 2 weeks \\
\hline Case 7 & $230.0 \mathrm{ng} / \mathrm{ml}$ (0 week) & $150.2 \mathrm{ng} / \mathrm{ml}$ (2 weeks) & $34.7 \%$ & 3.5 weeks \\
\hline Case 9 & $78.3 \mathrm{ng} / \mathrm{ml}$ (0 week) & $71.7 \mathrm{ng} / \mathrm{ml}$ (8 weeks) & $8.4 \%$ & II weeks \\
\hline
\end{tabular}

*I CEA level just before the decrease, *2 Period after the first vaccination, *3 (Baseline level - Lowest level) / Baseline level $\times 100(\%)$

included severe nausea, loss of appetite, and gastritis. Case 9 had been treated with CPT-11 (40 mg/day) once a week and 5'-DFUR (800 mg/day) every day for a year as adjuvant chemotherapy. After recurrence, all patients were treated with peptide vaccination without additional chemotherapy. Interestingly, the patients who showed no decrease in tumor markers received additional chemotherapy after recurrence and prior to vaccination. It is possible that the additional chemotherapy might have interfered with the vaccine.

Among four patients who were defined as NC or MR, two (case 1 and case 3 ) experienced decreases in tumor markers while the other two (case 11 and case 17) did not. In case 11 , levels of CEA have stayed within the normal range (0 5.9 $\mathrm{ng} / \mathrm{ml}$ ) until the 4th vaccination and increased thereafter (Fig. 2A). while CA19-9 levels stayed within the normal range $(0 \sim 37 \mathrm{U} / \mathrm{ml})$ throughout the study (Fig. 2B).

Case 1 was a 63-year-old man who had advanced rectal cancer. He underwent low anterior resection, followed by receiving tegafur/uracil (UFT) at a daily dose of $600 \mathrm{mg}$ as adjuvant chemotherapy for one year. At his own request, administration of the drug was discontinued. Three months later, he self referred himself with pygalgia. CT scan imaging demonstrated one lung metastasis, a tumor in the pelvis and a mass in the urinary bladder. Cystoscopy revealed that the bladder lesion was related to prostate hypertrophy while colonoscopy confirmed recurrent tumor in the pelvic cavity. The primary rectal cancer was confirmed by immunohistochemical staining to express survivin and the vaccination was started in March 2003. CEA and CA19-9 were $15.9 \mathrm{ng} / \mathrm{ml}$ and $152.4 \mathrm{U} / \mathrm{ml}$ respectively before treatment. They decreased continuously until the $5^{\text {th }}$ vaccination, at which points CEA and CA19-9 reached lowest levels, $6.5 \mathrm{ng} / \mathrm{ml}$ and $64.2 \mathrm{U} / \mathrm{ml}$, respectively. After the $6^{\text {th }}$ vaccination, however, they returned to increase to $16.2 \mathrm{ng} / \mathrm{ml}$ and $128.9 \mathrm{U} / \mathrm{ml}$, respectively (Fig. $3 \mathrm{~A}$ and $3 \mathrm{~B})$. After the 4 th vaccination, CT scan imaging revealed $32 \%$ reduction in volume of the recurrent pelvic tumor (Fig. 4A and 4B) while rectal bleeding disappeared after the $2^{\text {nd }}$ vaccination.

\section{DTH skin test}

DTH skin test was performed at each vaccination and assessed $48 \mathrm{hr}$ later. A positive reaction was defined as erythema and induration of more than $4 \mathrm{~mm}$ diameter. In 6 out of 15 patients $(40 \%)$, a positive DTH reaction was observed at least once during the study.

\section{Tetramer staining assay and ELISPOT assay}

In order to examine if the survivin-2B peptide vaccine could bring about a specific immune response in the patients, we analyzed the peptide-specifc CTL frequency by using HLA-A24/peptide tetramer. Survivin-2B peptidespecific CTL frequencies in PBMCs remained at background levels (less than $0.1 \%$ ) in all cases except for case 17. In case 17 , the frequency of tetramer-positive CTL was increased from $0.09 \%$ to $0.35 \%$ after the 4 th vaccination (Fig. 5). ELISPOT assay of CD8-positive T cells separated from the patients' PBMCs failed to show the peptide-specific IFN- $\gamma$ response in any patients (data not shown). Overall this study suggests that the vaccination approach that we attempted may provide marginal if any immunological or clinical benefit. However, it is possible that further studies that may include a more intense immunization schedule, delivery in the presence of adjuvant and/or the combination of immune stimulatory cytokines may enhance the activity of survivin as an immunogen against cancer.

\section{Discussion}

Recently, a large number of tumor antigens and epitopes recognized by cytotoxic T lymphocytes (CTLs) have been identified, and reports of clinical trials utilizing peptide vaccination are increasing [2,14-16]. However, most clinical trials were aimed at the treatment of advanced melanoma, and there are few reports of clinical trials for patients with solid gastrointestinal tract cancer. Therefore, we attempted to identify tumor antigens that are expressed in a wide variety of solid gastrointestinal tract 


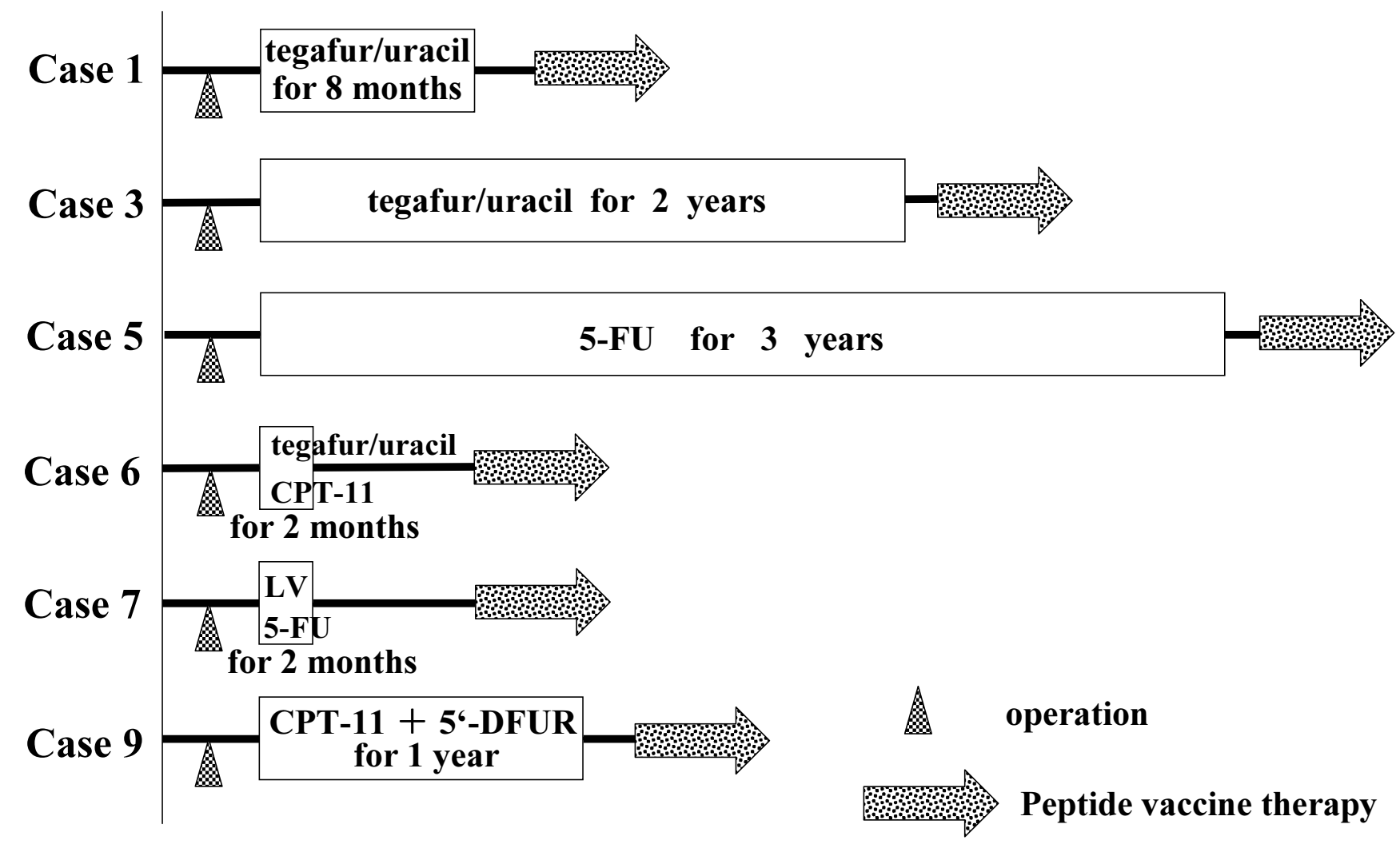

Figure I

Treatment history of six patients who experience decrease in tumor marker levels during immunization. Case I has received tegafur/uracil (UFT) at a daily dose of $600 \mathrm{mg}$ for eight months as oral adjuvant chemotherapy. Case 3 received tegafur/uracil (UFT) at a daily dose of $600 \mathrm{mg}$ for two years as oral adjuvant chemotherapy. Case 5 received 5-FU at a daily dose of $200 \mathrm{mg}$ for three years as oral adjuvant chemotherapy. Case 6 received 2 courses of CPT-I I (40 mg/day) every 4 weeks as adjuvant chemotherapy. Case 7 received 2 courses of leucovorin $(750 \mathrm{mg} /$ day $)$ and 5-FU (250 mg/day) every 4 weeks as adjuvant chemotherapy. Case 9 was treated with CPT-I I (40 mg/day) weekly and 5'-DFUR ( $800 \mathrm{mg} /$ day) for a year as adjuvant chemotherapy. They all had recurrences after the above adjuvant chemotherapy and received peptide-based vaccination without additional chemotherapy.

cancers. We demonstrated that survivin was expressed in a large proportion of colorectal and gastric cancer specimens, and the survivin-2B-derived peptide could induce CTL response in the context of HLA-A24 $[9,10]$. In addition, we showed an elevation in CTL precursor frequencies in PBMCs of HLA-A24+ cancer patients by using HLAA24/survivin-2B peptide tetramer. On the basis of the above studies, we started a phase I clinical study of survivin-2B peptide vaccine therapy for patients with advanced or recurrent colorectal cancer in March 2003. In many clinical trials, patients received the peptide in combination with certain adjuvant, such as IFA and cytokines, for the purpose of enhancing immune responses against cancer. In this study, patients received the survivin-2B 
Fig. 2A

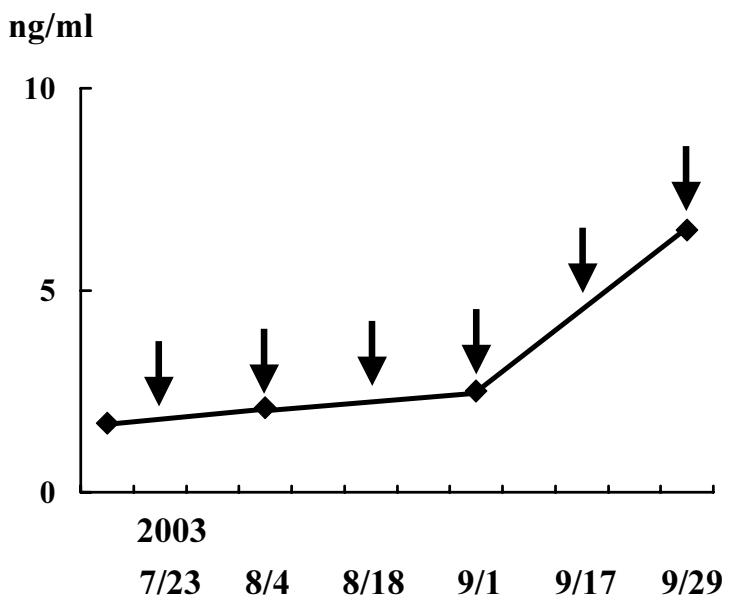

Fig. 2B

\section{$\mathbf{U} / \mathbf{m l}$}

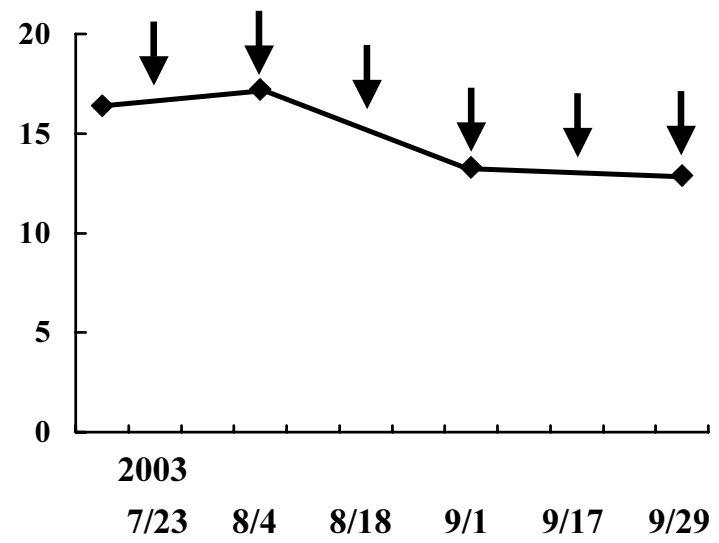

\section{Figure 2}

Changes in serum tumor marker levels during the study in case II (A: CEA, B: CAI9-9). Levels of CEA stayed within the normal range $(0 \sim 5.9 \mathrm{ng} / \mathrm{ml})$ until the 4th vaccination, and increased thereafter $(A)$. Levels of CAI9-9 stayed within the normal range $(0 \sim 37 \mathrm{U} / \mathrm{ml})$ throughout the study $(B)$. Arrows indicate the times of vaccination.

peptide dissolved in physiological saline without any adjuvant in order to strictly evaluate the clinical effect of the peptide alone.

A dose-escalation trial was chosen to estimate a safe and optimal dose. $0.1 \mathrm{mg}, 1.0 \mathrm{mg}$, and $10.0 \mathrm{mg}$ dosage groups, each consisting of five patients, were set up. Of the patients in the $0.1 \mathrm{mg}$ dose group, mild side effects were observed in two patients, such as general malaise (grade 1) and anemia (grade 2). None of the patients receiving $1.0 \mathrm{mg}$ had any sign of toxicity, and one of the patients in the $10.0 \mathrm{mg}$ dose group had slight fever (grade 1). No greater than grade 3 toxicity was observed. Thus, we conclude that the survivin-2B peptide vaccine is safe and can be repeatedly injected into patients without serious side effect. In addition, there was no correlation between toxicity grade and dosage within the range of $0.1 \mathrm{mg}$ to 10 mg.

In terms of clinical response, levels of tumor markers were temporarily decreased compared with pre-treatment in four patients in the $0.1 \mathrm{mg}$ dosage group and two patients in the $1.0 \mathrm{mg}$ dosage group. No patients in the $10.0 \mathrm{mg}$ dosage group experienced decrease in tumor markers. In 
Fig. 3A

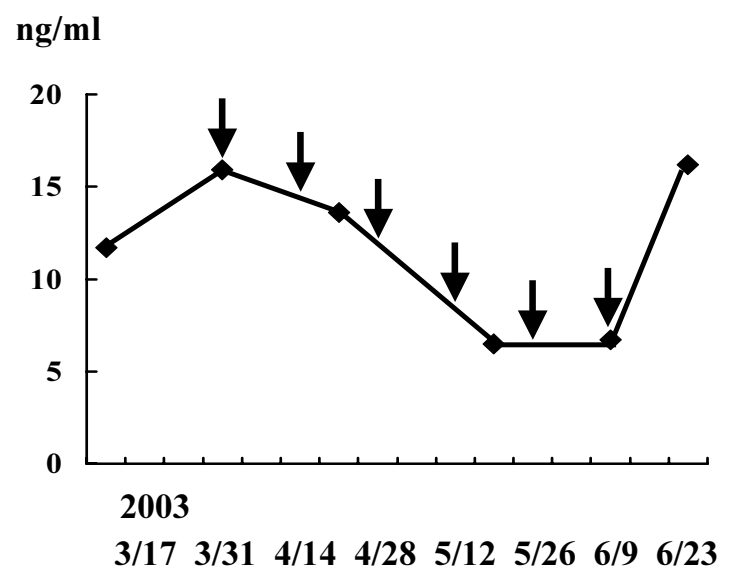

Fig. 3B

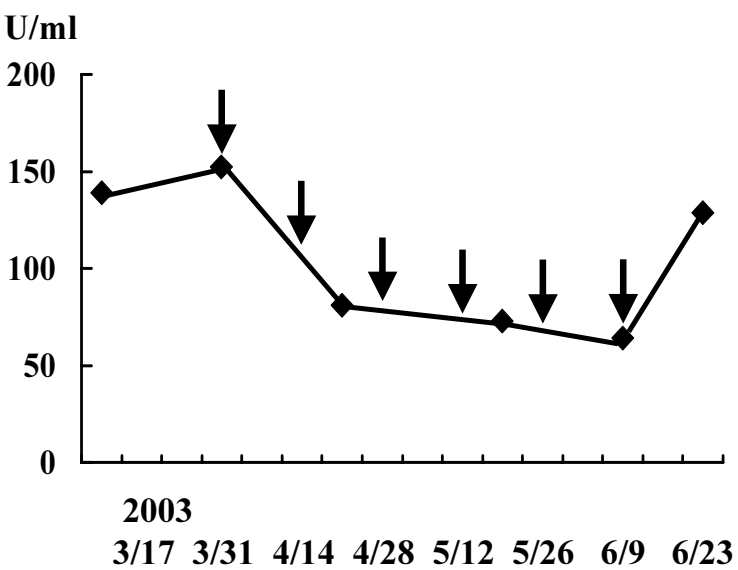

Figure 3

Changes in serum tumor marker levels during the study in case I (A: CEA, B: CA 19-9). Levels of CEA and CAI9-9 were I5.9 $\mathrm{ng} / \mathrm{ml}$ and $152.4 \mathrm{U} / \mathrm{ml}$, respectively before vaccination. Levels decreased continuously until the $4^{\text {th }}$ or $5^{\text {th }}$ vaccination to $6.5 \mathrm{ng} /$ $\mathrm{ml}$ and $64.2 \mathrm{U} / \mathrm{ml}$, respectively. However, they increased to $16.2 \mathrm{ng} / \mathrm{ml}$ and $128.9 \mathrm{U} / \mathrm{ml}$ respectively after $6^{\text {th }}$ vaccination.

Arrows indicate the times of vaccination.

addition, a minor response was observed in one patient who received $0.1 \mathrm{mg}$. Thus, there appeared to be no correlation between clinical outcome and vaccine dosage.

Positive DTH reaction was observed in 6 out of 15 patients $(40 \%)$ at least once during the vaccination. A previous report suggested a positive correlation between DTH and clinical responses [17]. In this study, however, neither level of tumor marker or tumor size were associated with positive DTH reaction, except for one case (case 6).

In this study, six patients (case 1, case 3, case 5, case 6, case 7, case 9) had temporary decreases in the level of tumor markers. It was noted that they had a history of adjuvant chemotherapy after operation, and no additional chemotherapy after recurrence while all the other patients received various kinds of chemotherapy after recurrence, prior to the vaccine treatment. Although our study consisted of only a limited number of patients, these preliminary data may suggest that peptide-based vaccines might be more effective in patients not immune-suppressed by recent chemotherapy.

Patient in case 17 had advanced rectal cancer and was operated on for creating a stoma without resection of the tumor. Though the levels of tumor markers continued to increase during the study, HLA-A24/peptide tetramer analysis of CD8+ T cells in PBMCs suggested a minor increase in frequency of peptide-specific CTL after the $4^{\text {th }}$ vaccination (approximately four-fold). It is possible that this the CTL response induced by the vaccine might not be sufficient to induce clinical regression in a patient with advanced or the tetramer-positive CTL might be suppressed in their cytotoxic function. Though immunologi- 
Fig. 4A

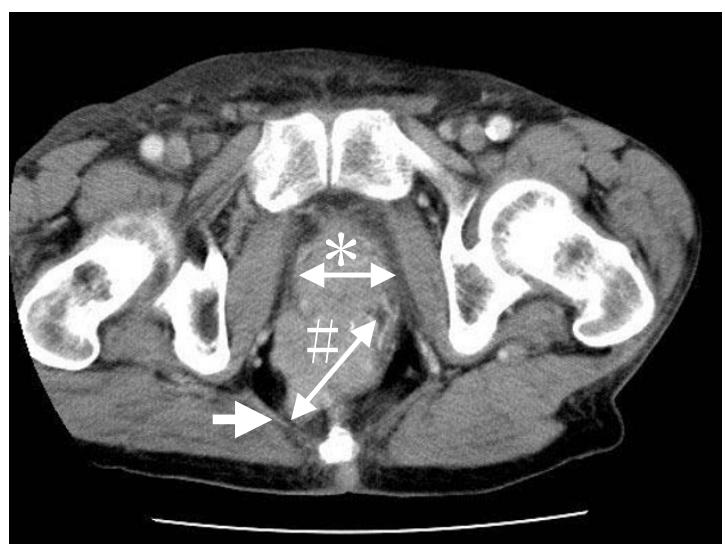

Fig. 4B

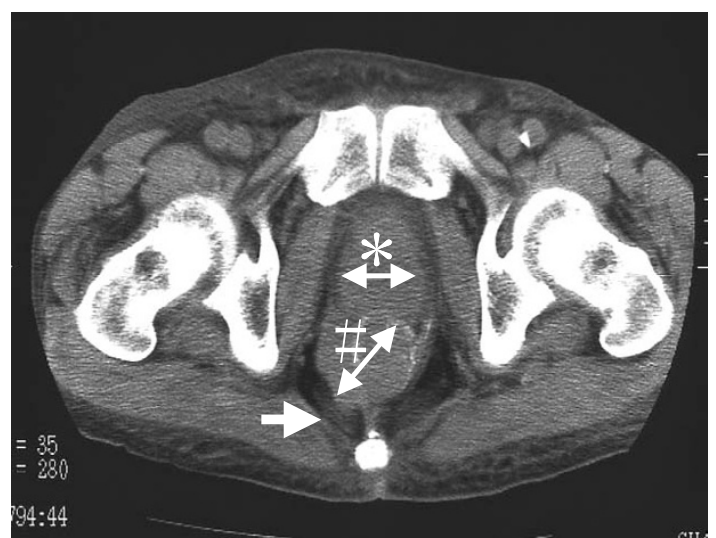

\section{Figure 4}

CT scan image of the pelvis of patient reported as case I (A: before vaccination, B: after the $4^{\text {th }}$ vaccination). The CT scan image demonstrates a reduction of tumor volume in the pelvic cavity after the $4^{\text {th }}$ vaccination compared with before vaccination (32\% reduction). The tumor had spread to the gluteus maximus muscle before treatment (white arrow in Panel A), whereas it appears separated from the muscle (white arrow in Panel B) after vaccination. The asterisk $(*)$ indicates prostate hypertrophy. The sharp-mark (\#) indicates a recurrent tumor in the pelvic cavity.

cal monitoring has been executed using tetramer assay in the other cases, tetramer-positive CTL frequencies were under the detection limits even after the $6^{\text {th }}$ vaccination. It is obvious that a sensitivity of the assay has to be increased to detect the specific CTL in patients' PBMCs. In some reports, patients' PBMCs were stimulated with peptides in the presence of IL-2 in vitro for several days before tetramer assay. In our present immune monitoring study, we examined the CTL frequencies without such in vitro expansion since it was anticipated that such an approach might not be representative of the naturally occurring immune response against the peptide in vivo.
This phase I clinical study revealed that the administration of the survivin-2B peptide is safe. It is obvious that superior clinical and immunological responses are necessary for cancer immunotherapy. Though vaccination with survivin-2B peptide alone was not enough to elicit overt clinical responses, it is possible that vaccination with peptide in combination with adjuvants or cytokines may be able to lead to higher immune responses both in the induction phase and in the effector phase. On the basis of the information obtained from this study, we have recently started the second clinical study of survivin-2B peptide vaccine in 
Fig. 5A

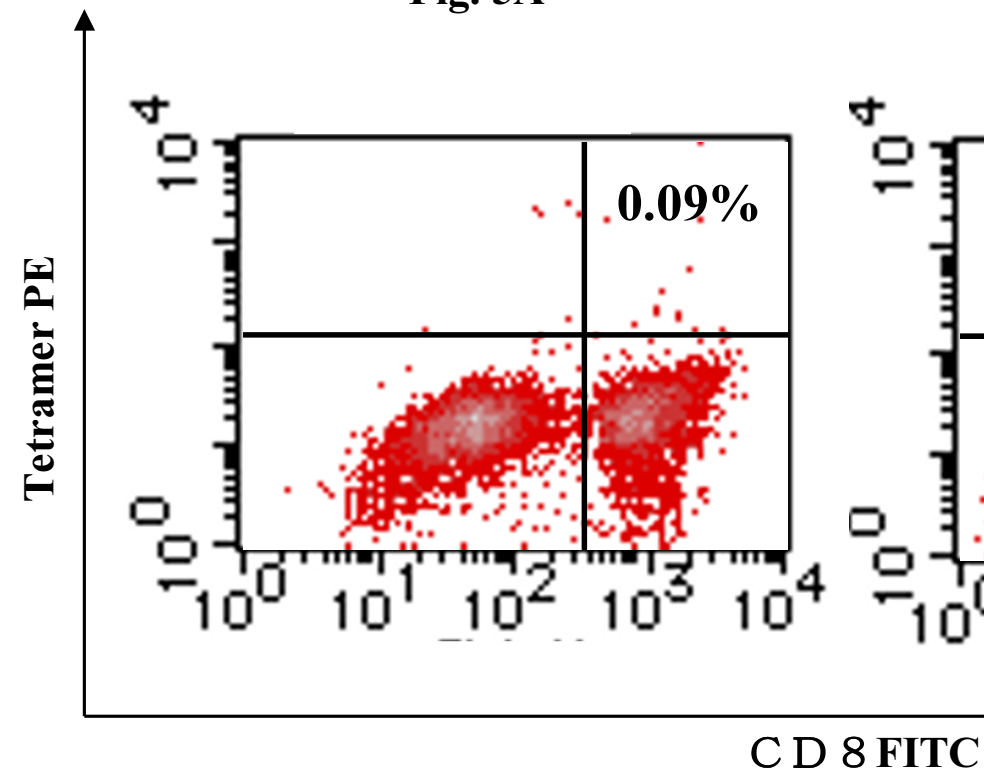

FIC
Fig. 5B

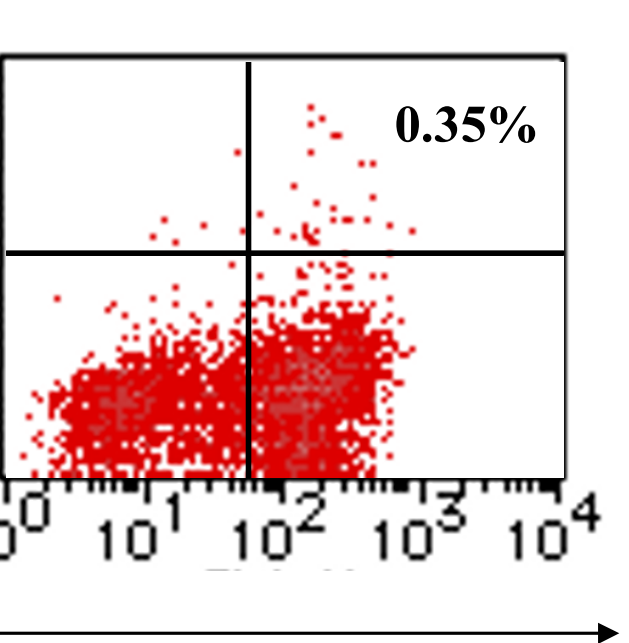

\section{Figure 5}

Tetramer analysis of PBMCs from case 17. Lymphocytes were collected from peripheral blood of the patient reported as case 17 before the $\left.\right|^{\text {st }}$ vaccination $(A)$ and after the $4^{\text {th }}$ vaccination $(B)$, stained with FITC-labeled antiCD8 mAb and PE-labeled HLAA24/survivin 2B peptide tetramer, and analyzed by flow cytometry. The frequency of tetramer-positive CTL was increased from $0.09 \%$ to $0.35 \%$ of CD8+ $T$ cells after the 4 th vaccination.

combination with various adjuvants such as IFA, GM-CSF, IL-2 and IFN- $\alpha$.

\section{Abbreviations}

CTL; cytotoxic T lymphocyte

IAP; inhibitor of the apoptosis proteins

TAAs; tumor-associated antigens

ECOG; Eastern Co-operative Oncology Group

DTH; delyed-type hypersensitivity
CR; complete response

PR; partial response

$\mathrm{NC}$; no change

PD; progressive disease

MR; minor response

IFA; incomplete Freund's adjuvant 


\section{References}

I. Zinkernagel RM, Doherty PC: Restriction of in vitro T cell-mediated cytootoxicity in lymphocytic choriomeningitis within a syngeneic or semiallogeneic system. Nature 1974, 248:70I-702.

2. van der Bruggen $P$, Traversari $C$, Chomez $P$, Lurquin $C$, De Plasen E, $V$ an den Eynde $B$, Knuth $A$, Boon $T$ : $A$ gene encoding an antigen recognized by cytolytic $T$ lymphocytes on a human melanoma. Science 1991, 254:|643-1647.

3. Traversari C, van der Bruggen $P$, Luescher IF, Lurquin C, Chomez $P$, Van Pel A, De Plaen E, Amar-Costesec A, Boon T: A nonapeptide encoded by human gene MAGE-I is recognized on HLA-A I by cytolytic $\mathrm{T}$ lymphocytes directed against tumor antigen MZ2-E. J Exp Med 1992, I 76: |453-| 457.

4. Kawakami Y, Eliyahu S, Delgado CH, Robbins PF, Rivoltini L, Topalian SL, Miki T, Rosenberg SA: Cloning of the gene coding for a shared antigen recognized by autologous $T$ cells infiltrating into tumor. Proc Natl Acad Sci USA 1994, 9 I:35I 5-35I9.

5. Parmiani G, Castelli C, Dalerba P, Mortarini R, Rivoltini L, Marincola FM, Anichini A: Cancer immunothrapy with peptide-based vaccines: what have we achieved? Where are we going? J Natl Cancer Inst 2002, 94:805-818.

6. Rosenberg SA, Yang JC, Schwartzentruber DJ, Hwu P, Marincola FM, Topalian SL, Restifo NP, Dudley ME, Schwarz SL, Spiess PJ, Wunderlich JR, Parkhurst MR, Kawakami Y, Seipp CA, Einhorn JH, White DE: Immunologic and therapeutic evaluation of a synthetic peptide vaccine for the treatment of patients with metastatic melanoma. Nat Med 1998, 4:321-327.

7. Ambrosini G, Adida C, Altieri DC: A novel anti-apoptosis gene, surviving, expressed in cancer and lymphoma. Nat Med 1997, 3:917-921.

8. Altieri DC.: The molecular basis and potential role of survivin in cancer diagnosis and therapy. Trends Mol Med 200I, 7:542-547.

9. Yoshihiko Hirohashi, Toshihiko Torigoe, Akiko Maeda, Yuki Nabeta, Kenjiro Kamiguchi, Takashi Sato, Junichi Yoda, Hideyuki Ikeda, Kouichi Hirata, Noboru Yamanaka, Noriyuki Sato: An HLA-A24restricted cytotoxic $T$ lymphocyte epitope of a tumor-associated protein, survivin. Clinical Cancer Research 2002, 8:173I-1739.

10. Satomi Idenoue, Yoshihiko Hirohashi, Toshihiko Torigoe, Yuriko Sato, Yasuaki Tamura, Hiroyuki Hariu, Masaaki Yamamoto, Takehiro Kurotaki, Tetsuhiro Tsuruma, Hiroko Asanuma, Takayuki Kanaseki, Hideyuki Ikeda, Kiyoteru Kashiwagi, Minoru Okazaki, Kazuaki Sasaki, Takashi Sato, Tosei Ohmura, Fumitake Hata, Koji Yamaguchi, Koichi Hirata, Noriyuki Sato: A potent immunogenic general cancer vaccine that targets survivin, an inhibitor of apoptosis proteins. Clinical Cancer Research in press.

II. Altman JD, Moss PA, Goulder PJ, Barouch DH, McHeyzer-Williams MG, Bell JI, McMichael AJ, Davis MM: Phenotypic analysis of antigen-specific T lymphocytes. Science 1996, 274:94.

12. Sato Y, Sahara H, Tsukahara T, Kondo M, Hirohashi Y, Nabeta Y, Kawaguchi S, Ikeda H, Torigoe T, Ichimiya S, Tamura $Y$, Wada T, Yamashita T, Goto M, Takasu H, Sato N: Improved generation of HLA class/peptide tetramers. J Immunol Methods 2002, 20(27 I (I-2)): I 77-I84.

13. Sato Y, Nabeta Y, Tsukahara T, Hirohashi Y, Syunsui R, Maeda A, Sahara $H$, Ikeda $H$, Torigoe T, Ichimiya S, Wada T, Yamashita T, Hiraga H, Kawai A, Ishii T, Araki N, Myoui A, Matsumoto S, Umeda $\mathrm{T}$, Ishii S, Kawaguchi S, Sato N: Detection and induction of CTLs specific for SYT-SSX-derived peptides in HLA-A24(+) patients with synovial sarcoma. J Immunol 2002, 169:1611-16 I8.

14. Yang D, Nakao M, Shichijo S, Sasatomi T, Takasu H, Matsumoto H, Mori K, Hayashi A, Yamana H, Shirouzu K: Identification of a gene coding for a protein possessing shared tumor epitopes capable of inducing HLA-A24-restricted cytotoxic T lymphocytes in cancer patients. Cancer Res 1999, 59:4056-4063.

I5. Nishizaka S, Gomi S, Harada K, Oizumi K, Itoh K, Shichijo S: A new tumor-rejection antigen recognized by cytotoxic $T$ lymphocytes infiltrating into a lung adenocarcinoma. Cancer Res 2000, 60:4830-4837.

16. Gjertsen MK, Buanes T, Rosseland AR, Bakka A, Gladhaug I, Soreide O, Eriksen JA, Moller M, Baksaas I, Lothe RA, Saeterdal I, Gaudernack $\mathrm{G}$ : Intradermal ras peptide vaccination with granulocytemacrophage colony-stimulating factor as adjuvant: Clinical and immunological responses in patients with pancreatic adenocarcinoma. Int J Cancer 2001, 92:44I-50.
17. Nestle FO, Alijagic S, Gilliet M, Sun Y, Grabbe S, Dummer R, Burg G, Schadendorf $D$ : Vaccination of melanoma patients with peptide- or tumor lysate-pulsed dendritic cells. Nat Med 1998, 4:328-332.
Publish with Biomed Central and every scientist can read your work free of charge

"BioMed Central will be the most significant development for disseminating the results of biomedical research in our lifetime. "

Sir Paul Nurse, Cancer Research UK

Your research papers will be:

- available free of charge to the entire biomedical community

- peer reviewed and published immediately upon acceptance

- cited in PubMed and archived on PubMed Central

- yours - you keep the copyright

Submit your manuscript here:

http://www.biomedcentral.com/info/publishing_adv.asp
BioMedcentral 